\title{
Poster Sessions
}

for $\mathrm{LuBO}_{3}$ in process of annealing of the amorphous precursors: high vaterite phase $\rightarrow$ calcite phase $\leftrightarrow$ high vaterite phase again instead of the previously known sequence: calcite phase $\leftrightarrow$ high vaterite phase. For $\mathrm{LaBO}_{3}$ instead of sequence: aragonite phase $\leftrightarrow$ monoclinic phase in micro dispersed powders we have the sequence: monoclinic phase $\rightarrow$ aragonite phase $\leftrightarrow$ monoclinic phase if the crystallization is realized from amorphous precursor.

The third phenomenon consists in acceleration of the phase formation at lower temperature and for shorter time if temperature of the sample under synthesis is increased continuously. Such effect was observed in process of borates $\mathrm{RBO}_{3}$ and garnets $\mathrm{R}_{3} \mathrm{M}_{5} \mathrm{O}_{12}$ synthesis. It was established that the initiating effect of the continuous heating on synthesis of the garnets, which have cubic structure for all R-atoms only, is realized in more rapid growth of the crystallites from nanoto micro-sizes. For the borates which have a few different phases in dependence of R-atom used the continuous heating brings to formation together with equilibrium phase the new phases known for other rare earth elements.

The fourth phenomenon is the effect of "structure infection". Such effect was observed at first stages of $\mathrm{YBO}_{3}$ crystallization after adding a few percents of $\mathrm{Sc}$ atoms in amorphous precursor. In this case calcite phase of $\mathrm{YBO}_{3}$ is formed. However it is unknown for yttrium borate but is stable phase for $\mathrm{ScBO}_{3}$. Almost the same situation is observed in the process of $\mathrm{YAlO}_{3}$ perovskite phase synthesis. The hexagonal phase of $\mathrm{YAlO}_{3}$ can to form at first steps of crystallization (T $\sim 600 \mathrm{C}$ ) after adding a small amount of $\mathrm{La}_{2} \mathrm{O}_{3}$ (hexagonal structure) in amorphous precursor while such phase is known in micro-dispersed state at $\mathrm{T}>1400 \mathrm{C}$ only.

Keywords: nanocrystal, rare-earth compound, X-ray

\section{MS49.P06}

Acta Cryst. (2011) A67, C534

Improvement of crystal qualities by solution stirring techniques Mihoko Maruyama, ${ }^{\text {a }}$ Kosuke Murakami, ${ }^{\text {a }}$ Yoshinori Takahashi, ${ }^{\mathrm{a}}$

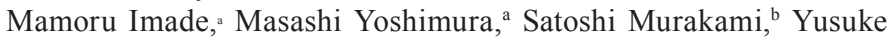
Mori, ${ }^{a}{ }^{a}$ Graduate School of Engineering, Osaka University, ${ }^{b}$ Department of Life Science, Tokyo Institute of Technology. E-mail: maruyama@cryst.eei.eng.osaka-u.ac.jp

Solution stirring techniques are effective to make crystals with high quality, and widely introduced in industrial field. We have succeeded in growing high quality $\mathrm{CsLiB}_{6} \mathrm{O}_{10}$ and DAST by utilizing solution stirring techniques. ${ }^{\text {The }}$ Thesults show that availability of the stirring techniques covers not only inorganic materials but also organic materials. In this presentation, we will introduce effects of solution stirring on $\mathrm{GaN}$ crystals and some protein crystals (hen-egg white lysozyme (HEWL) and glucose isomerase (GI)). Comparison of these results will show some mechanisms how the solution stirring techniques improve the crystal qualities.

We introduced sodium flux method to grow GaN single crystals. Using this method, GaN can be grown by the dissolution of pressurized nitrogen gas into Ga-Na melt under conditions of approximately $\sim 1143 \mathrm{~K}$ and 3.4 MPa. Growth condition easily becomes unhomogeneous in a crucible, then some problems such as unfavorable nucleation, nonuniform crystallization on templates and low yield happen. We must comprehend and control solution conditions to reduce these problems. As a first step we investigated a condition in a crucible. To know the condition distribution in the crucible, we grew some of GaN crystals concentrically in the crucible. A main parameter was solution stirring, that is (1) no stirring, (2) stirring by rotation of a chamber, (3) stirring by fluctuation of a chamber. Sizes, morphologies and surface conditions of these grown crystals reflect a distribution of the supersaturation. As a result, both of the stirring techniques effectively suppress unfavorable nucleation and achieve faster growth rate than no stirring system. Furthermore solution stirring improved polycrystals and skeletal crystals. These results are obvious evidences for homogenized solution conditions.

In the field of crystallography of protein crystals, crystallographers must consider many conditions and usually use a protein solution of less than $10 \mu$ in each batch. Thus we applied specially designed rotary shaker to introduce stirring in protein crystal growth. Crystallization plates were set on the rotary shaker, and stable solution flow arose in each batch. ${ }^{3}$ In the case of protein crystal growth, surface kinetics can be a late-determining process in many cases, because of the difficulty of accurate molecules rotation and incorporation into crystals. Actually shapes of crystals with or without stirring were almost same in both the case of HEWL and GI. However, densities of defects were strongly affected by solution stirring. The defects densities were estimated by crystal etching. Two kinds of etch pits on HEWL and GI crystals were observed after etching. One is a shallow pit, which indicates the micro defects such as impurities or holes, ${ }^{4}$ and the other is deep pit, which indicates the dislocations in crystals. The shallow pits' density tended to decrease with stirring speed. This tendency implies that transportation of impurities, which can be the micro defects, to crystal surface is suppressed by solution stirring. We have succeeded to improve the quality and size of some protein crystals by introducing the stirring method. ${ }^{5}$ The decrease of shallow pit density probably is a contribution factor for crystal quality improvement.

As seen above, the way to stir solution and main effects of stirring depend on materials. In the case of $\mathrm{GaN}$ crystals, stirring contributed to uniformize solutions in crucible, and in the case of protein crystals, the decrease of micro defects was a main effect of stirring. However, we would like to emphasize that solution-stirring techniques finally improved crystal qualities. Solution stirring is one of a universal principle that is applicable to various materials.

[1] Sasaki et al., J.Cryst. Growth, 2008, 310 1288. [2] Yamane et al., Chem. Mater. 1997, 9, 413. [3] Adachi et al., Jpn. J. Appl. Phys. 2003, 42, L314. [4] Hondoh, et al, Jpn, J. Appl. Phys. 2005, 43, 4529. [5] Adachi, et al, Jpn. J. Appl. Phys. 2003, 42, L314.

Keywords: solution stirring, GaN, protein crystal

\section{MS49.P07}

Acta Cryst. (2011) A67, C534-C535

Mechanical property measurements of growing lysozyme crystal by atomic force microscopy with laser confocal differential interference microscopy

Shin-ichiro Yanagiya and Nobuo Goto, Department of Optical Science and Technology, the University of Tokushima, (Japan).E-mail: giya@, opt.tokushima-u.ac.jp

We have studied combination of optical microscopy (OM) and atomic force microscopy (AFM). One of the combinations was the seamless observation of OM and AFM [1]. We observed the surface of a potassium dihydrogen phosphateIn (KDP) crystal by transmission optical microscopy, the laser confocal differential interference microscopy (LCM-DIM, [2]), and AFM. In that study, the LCM-DIM played important role as an intermediary because of its high z-axis resolution. Another study was about the interferometric microscopy using AFM cantilever. We demonstrated the observation of growing steps with measuring the step height [1]. In addition, we also demonstrated measurement of refractive index of pure water by measuring the distance between the interference fringes [3]. The point was its measurement area, which was about 10-micrometer-square region. In this study, we focused on measurements of mechanical 